\title{
IDENTIFICATION OF NONLINEAR MICRON-LEVEL MECHANICS FOR A PRECISION DEPLOYABLE JOINT
}

\author{
S. J. Bullock " and L. D. Peterson ${ }^{\dagger}$ \\ Center for Aerospace Structures \\ and Department of Aerospace Engineering Sciences \\ University of Colorado at Boulder \\ Boulder, CO 80309-0 429
}

\begin{abstract}
The experimental identification of micron-level nonlinear joint mechanics and dynamics for a pin-clevis joint used in a precision, adaptive, deployable space structure are investigated. The force-state mapping method is used to identify the behavior of the joint under a preload. The results of applying a single tension-compression cycle to the joint under a tensile preload are presented. The observed micron-level behavior is highly nonlinear and involves all six rigid body motion degrees-of-freedom of the joint. It also suggests that at micron levels of motion modelling of the joint mechanics and dynamics must include the interactions between all internal joint components, such as the pin, bushings, and the joint node.
\end{abstract}

\section{INTRODUCTION}

Current research in reconfigurable, adaptive structures is focusing on increasing the mechanism's reliability and predictability. For precision structures, an additional concem is the behavior of joints on a micron level, as this has a direct impact on the operational precision of the structure. Detailed component modeling is one part of enhancing reliability, because good models lead to good confidence bounds on the systems performance. Proper simulation of the deployment process requires component models with the correct inertial, kinematic, load transmission, stiffness, and damping properties. Experimental identification of component models is essential because much of the nonlinear joint mechanics depends on heterogeneous mechanical properties, such as friction and contact mechanics, the effect of which can only be measured in situ.

This paper uses the force-state mapping (FSM) method to derive nonlinear micron-level component models for these types of applications. The FSM technique is a subset of a class of nonlinear identification techniques called restoring force methods. The first type of restoring force method was developed by Masri and Caughey [1]. The FSM method was independently developed by Crawley and Aubert [2], at a later date. Masri, Sassi, and Caughey [3] extended their technique to multiple degree-of-freedom

\footnotetext{
- Graduate Research Assistant, Student Member AIAA

t Assistant Professor, Senior Member AlAA, Associate Member ASME
}

Copyright 1994 by S. J. Bullock and L. D. Peterson. Published by the Anencan Institute of Aeronautics and Astronautics, Inc. witl permission.
(MDOF) systems, but did little experimental validation. Autcit Crawley, and O'Donnell [4] used FSM to identify single degrece of-freedom (SDOF) joints in flexible space structures. Master and Crawley [5] experimentally verified the FSM technique for MDOF systems. Worden and Tomlinson [6-7] have done much work in experimentally identifying nonlinear SDOF, MDOI: and time-dependent systems using restoring force methods

The assessment of the micron-level behavior of a family of precision pin-clevis joints while under a tensile preload through the use of FSM is the principle contribution of this research.

A class of joints common to many adaptive, deployable struc tures is the pin-clevis joint, as shown in Figure 1. This lype of joint does not contain any latching or preloading compencml This particular type of pin-clevis joint includes two internal com ponents (bushings), which cannot be seen in Figure 1. It is gec1. erally described as a SDOF joint because it only allows litrect motion about a single axis.

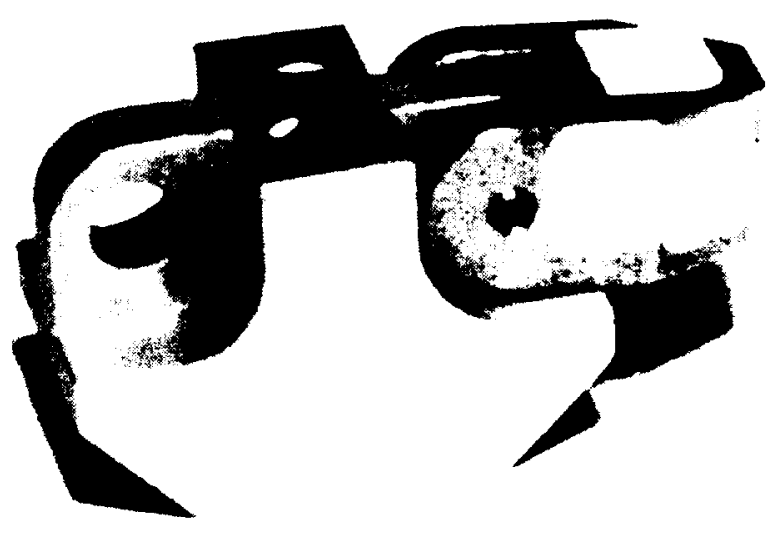

Figure 1: Pin-Clevis Joint Component Suhsystem

A parametric study of the static behavior of pin-clevis forint by Rhodes [8] gives many design recommendations for inakin! joints with high torsional and axial stiffness, increasing the rithice of their linear static behavior. However, this study did not int dress the dynamic behavior of pin-clevis joints. Nonline arituc m the dynamic behavior can be attributed to several charactetivic 
common to all classes of articulating joints: geometric nonlinearities due to the kinematics of large motion, nonlinearities due to the change in the load path when the joint experiences compressive and tensile forces, and joint free-play.

The geometric nonlinearities due to the kinematics of large motion manifest themselves in the inertia, damping, and stiffness tensors of the joint. Previous FSM investigations have exploited the fact that the system tensors are tangentially constant over a small range of motion. However, it becomes significantly dependent on the motion and position of the system when the joint rotations or motions become kinematically large. Restoring force methods can theoretically model any nonlinearity, as long as the inertia tensor is not directly a function of time. This study will also assume the system tensors are constant over a small range of angular motion.

The previous assumption will isolate a major cause of nonlinearities in pin joints, which is the change in the load path under compression and tension. From the theory of continuum mechanics, the localized effect of a circular hole in a plate is that the local tensile stress is three times greater than the uniform stress applied to the ends of the plate [9]. Although the node to which a pin joint is connected has a circular hole to accommodate the pin, it cannot be considered a plate because a significant portion of the "plate" on one side has been removed to allow the joint to rotate. When the joint is under tensile forces, the pin is constrained by a thin band of material. This band splits and then bends the tensile force vector back towards the center of the node. When the joint is under compressive forces, the pin transfers the force vector directly into the node center. In this situation, the stress concentration due to the circular hole will be analogous to the classical elasticity problem. Since the load path is different under compression and tension, the intuitive conclusion is that the joint behavior will differ under these two loading conditions, and therefore be nonlinear.

An additional factor affecting the load path is the location and nature of internal joint components. These can include bearings, bushings, surface coatings, and material hardening processes. The contact mechanics and compliance between internal components can greatly change the load path behavior from that found in an assembly that only has a joint, pin, and joint node.

The standard practice to eliminate the nonlinearities caused by joint free-play is to preload the joint in tension or compression. This theoretically should force the joint to behave linearly under all static and dynamic loads that do not cancel the effect of the preload. This work investigates the above assumption for motion on the micton level.

To study the micron-level dynamics of a joint under a tensile preload, this paper presents the experimental FSM identification of a simple, pin-clevis joint. A preliminary experiment to identify the behavior of a cantilevered beam is used to validate the experimental and data analysis protocols. The joint studied in this paper is one of the joints used in a batten-actuated deployable truss, shown in Figure 2. An investigation of the dynanic hehalyior of the entire truss linds a high degree of monlincarity due on the combined effects of all the pin-clevis joints $|\mathrm{l} 0|$

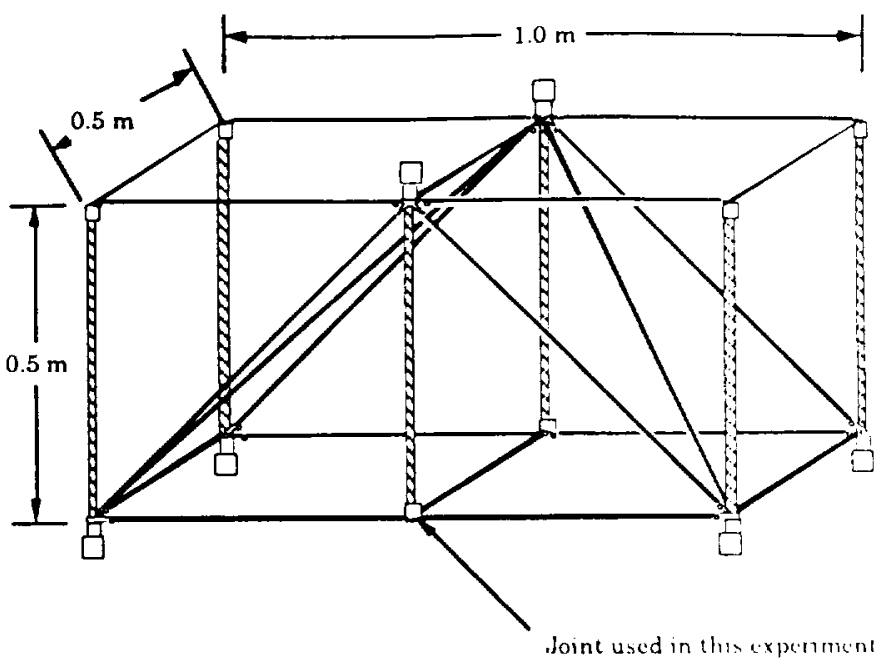

Figure 2: Reconfigurable Truss in Deployed state

The remainder of this paper is organized as lollows. In the first section, we detine restoring force methods atnd the f:SM technique. Next, we describe the deployable jonnt and imsumentation used in the experiment. The data collection prexedure is then outlined. The following sections definc the lixcel cxperimental parameters, describe the experimental protocol, and then outline the analysis protocol. In the last section, the expertincmitl results are reported and a qualitative analysis of the innt bchaior is made.

\section{RESTORING FORCE METHODS}

Restoring force methods assume that a physical. dynamic system obeys Newton's second law of motion for mass that is time invariant. For a SDOF system, the equation of motion (EOM) is given by

$$
m x(t)+f(x(t), x(t))=u(t)
$$

where $m$ is the mass of the system, $u(f)$ is an exlculally applicel force, $x(t)$ is the displacement of the mass, $x(t)$ is 11 siclecily, and $\ddot{x}(t)$ is its acceleration. The system mass ciul not be a tircel function of time, but can be indirectly time dependert thrugh dependence on the physical states. The lunction / is a genctid function of the internal or restoring force of the system So it the acceleration and external force are measured, the restoring lorci can be determined by

$$
f(x(t), x(t))=n-m x
$$

The measured restoring force can be visuadized at a surtice over the phase, or state, plane. This plot is cilled the toret-rtalc map of the system. 
The restoring force method developed by Masri and Caughey [1] generated a model for $f$ by fitting the measured data to a double expansion in Chebyshev polynomials in the variables $x(t)$ and $x(t)$. Crawley and Aubert [2] used a general polynomial expansion to model $f$ when they independently developed the force-state mapping method. The usual procedure for determining the coefficients of either type of expansion is by a direct least-squares method. The work by Al-Hadid and Wright [1112] has shown that general polynomial expansions produce better results than Chebyshev expansions, which is the reason this work uses the FSM method.

The true strength of restoring force methods is their ability to fit experimental data to models of physical phenomena. Systems with discontinuities in their stiffness or damping behavior $[4,6]$, hysteretic systems [6], and systems with time varying stiffness and damping properties [6] have all been experimentally identified. Restoring force methods can also accurately identify Coulomb friction $[4,6]$ and piecewise linear stiffness behavior [6]. When the behavior due to these physical phenomena is presented as a force-state map, a qualitative assessment of the influence of each type of phenomenum can be quickly made due to the unique graphical features characteristic of each phenomenum.

\section{TENSILE PRELOAD BEHAVIOR FORCE-STATE MAPPING EXPERIMENT}

The precision pin-clevis joint studied in this paper is one of several joints used in a deployable truss testbed being studied at the University of Colorado. The testbed has four different types of joints, one of which is shown in Figure 1. A top view of the cross-section of the joint and joint node is illustrated in Figure 3. The bronze bushings were chosen to be the bearing surface for both the joint and pin to avoid a costly hardening process of the adjoining surfaces of the aluminum joint body. The complete component subsystem is attached to a test article mounting interface, which in turn is mounted to a large backstop (see Figure 4).

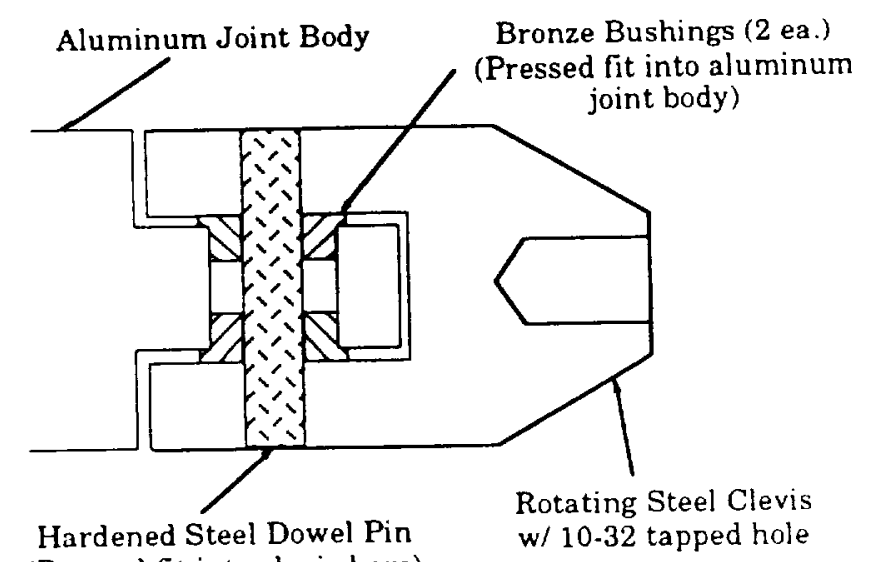

(Pressed fit into clevis here)

Figure 3: Cross-Section of Joint Assembly

Due to the size and mass $(25 \mathrm{~g})$ of the pin-clevis joint, a noncontacting sensor system was desirable. This was not possible for the measurement of the extemal force. The excitation force is provided by an electrodynamic shaker, which is also atlached 111 the backstop. A steel stinger from the shaker connects direclly to a force transducer, which in turn is attached to the joint at the point that normally is used for strut connections. The rest of the sensor system is composed of diree Keyence lasers, which provide direct displacement measurements of three laces of the joint. A top view of the joint and instrumentation is diagrammed in Figure 4, except the vertical laser head has been moved allu for the sake of clarity, and a picture of the FSM experiment is shown in Figure 5. The velocity and acceleration of the jom and obtained by differentiating the displacement measurements.

Backstop

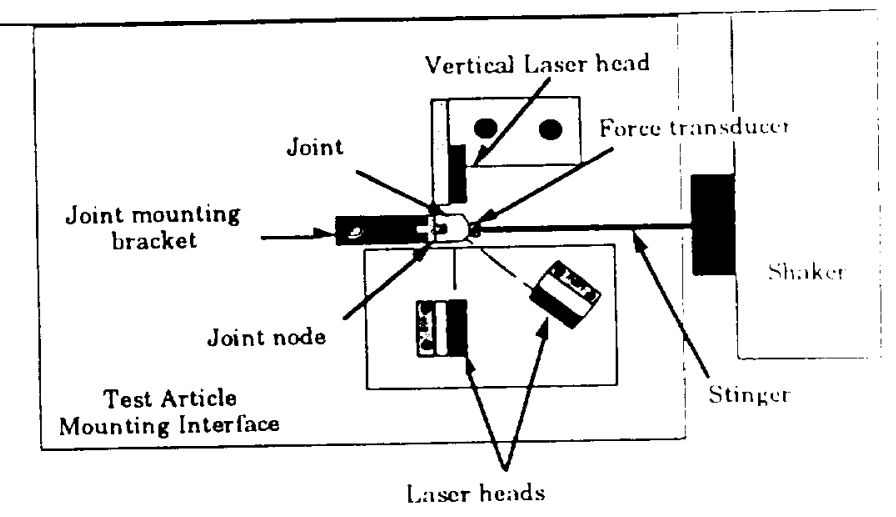

Figure 4: Top View of Test Configuration

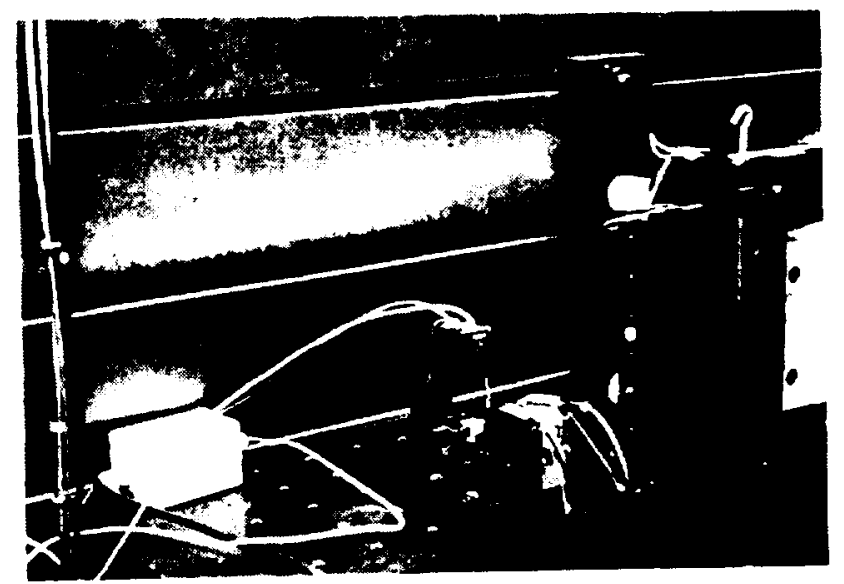

Figure 5: FSM experiment and instrumentation

An experiment to validate the testing and andysis protocols was performed on a cantilevered aluminum beiun, shown in ligure 6 . The beam is $4.76 \mathrm{~mm}$ drick, $22.0 \mathrm{~mm}$ wide, and $38.1 \mathrm{~mm}$ long. Bolts are used to join the various parts of the bean tcst illticle together. The distance between the centers of the holl hold on the beam is $28.6 \mathrm{~mm}$. The beam was sized to allow $25 \mathrm{~mm}$ crons of displacement at the tip under a transverse had of $45 \mathrm{~N}$ Half of a pin-clevis joint was attached to the lip of the bean wh provide the lasers with similar target locations as on the istual pin-clevis joint. This meant that the exact siunc antyly proleket could be used for both experiments to extrict the femsle-comn. 
pressive motion from the three laser measurements.

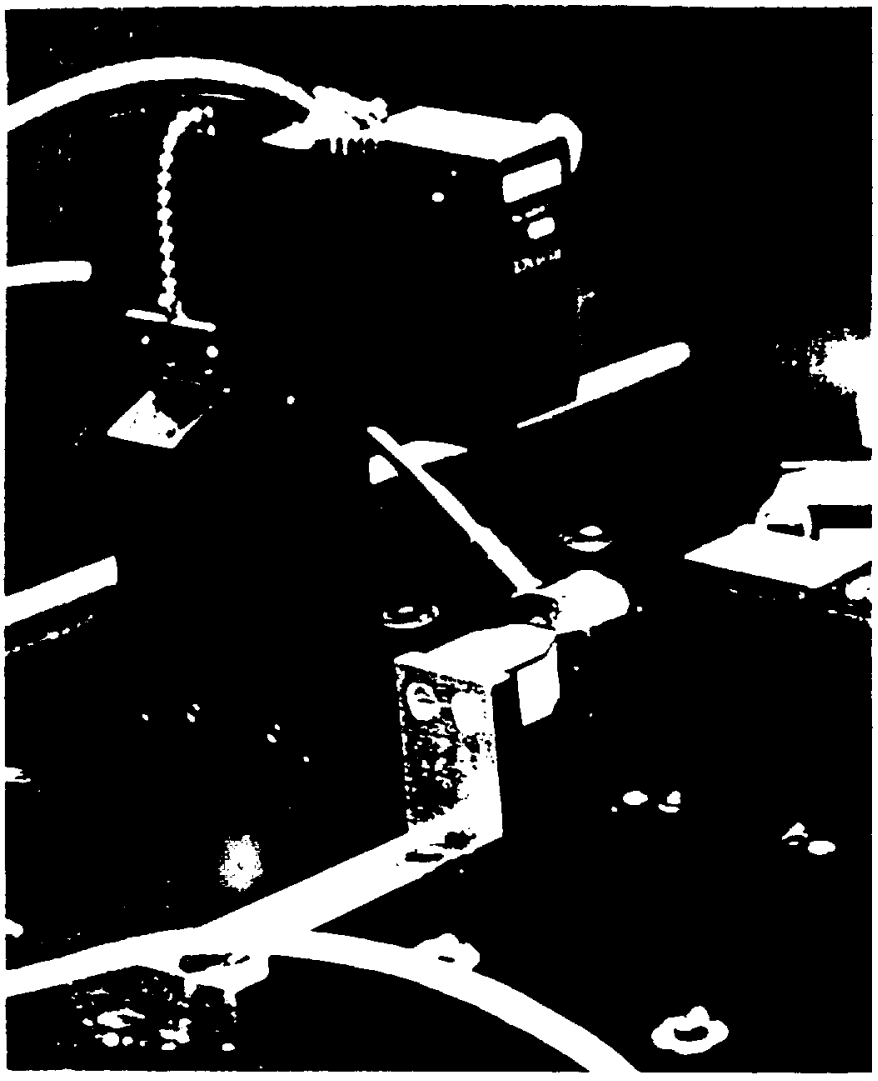

Figure 6: Cantilevered Beam Test Article

\section{Data Collection Protocol}

The equipment used to perform the experiment is diagrammed in Figure 7. A master program communicates to all equipment through a GPIB interface and a multi-channel input and output data acquisition board. A waveform generator program passes the waveform for a specific trial to the power amplifier. A gain multiplier for the waveform is manually set on the power amplifier. The amplified signal is then delivered to the shaker.A data collection program loads previously defined experimental parameters. Next, the data collection program initializes the data acquisition board and the simultaneous track-andhold module. Then the sensor gains and filter levels are set on a DC signal conditioner. The lasers and the force transducer send their signals to the DC signal conditioner during a trial. The signals are amplified and filtered by an eighth order Butterworth low-pass filter. A track-and-hold module simultaneously samples up to 8 channels, which are digitized by the 16-bit ADC on the data acquisition board. The filtered and digitized raw time data from each sensor is then recorded by the data collection program. A data analysis program performs additional data processing before generating FSM plots.

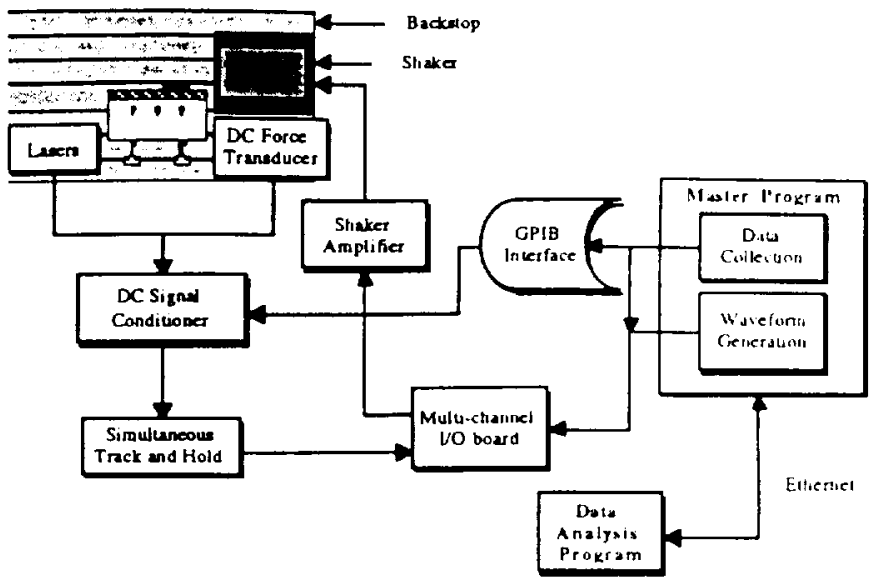

Figure 7: Hardware Configuration

\section{Experiment Parameters}

The parameter choices for this study are shown in Table I and are explained below.

Table 1: Experimental parameters

\begin{tabular}{|l|l|}
\hline Bandwidth of Interest & $0-100 \mathrm{~Hz}$ \\
\hline Sample Rate & $2000 \mathrm{~Hz}$ \\
\hline Sampling Window & -4 seconds \\
\hline Waveform & 1 cycle $0.31 \mathrm{~Hz}$ Sine wave \\
\hline Force Amplitude & $70 \mathrm{~N}(-15.7 \mathrm{lbf})$ \\
\hline Tensile Preload & $220 \mathrm{~N}(-49.5 \mathrm{lbf})$ \\
\hline
\end{tabular}

The bandwidth of interest was driven by the effective bandwidth of the lasers. The sample rate was chosen to provide a smooth, continuous digitized signal of the highest frequency of interest. This helped to minimize the errors introluced by differentiating the displacement measurements to obtain velocily and acceleration data. The sampling window length was a compromise between frequency resolution and data processing constraints due to large data sets.

The excitation signal selected was a single cycle of a $0.31 \mathrm{ll}$, sine wave. The sine wave signal had an anplitude of $1.00 \mathrm{Volls}$ and a 3.00 Volts offset. This produced the simplest excitation input signal that could maintain tension in the joint while the jom was loaded by a tensile-compressive cycle. $\wedge$ polentioncter on the shaker amplifier provided the scaling necessiry io converl the analog signal to a shaker force history with the chatracteristics listed in Table 1.

\section{Experimental Procedure}

The FSM experiment was designed to determine the force. state map for the tensile-compressive degree-of-trecdom of it simple, pin joint under a tensile preload. Since the system incrtlat. 
damping, and stiffness tensors are dependent on the rotational state of the joint, the testing procedure must be carefully designed to isolate this dependence when trying to identify the tensile-compressive behavior. The testing protocol assumes that gravity effects are negligible in the joint mechanics of the reconfigurable truss.

The influence of the rotational state was limited by assuming that the system tensors were tangentially constant for small angle motions. Therefore the testing protocol had to load the joint in such a way as to minimize angular motions due to compliance in the joint and due to rotation about the hinge line. This was accomplished by using a preload and a stiff steel stinger.

The experiment involving the cantilevered beam (see Figure 6) was designed to show that the experimental and analysis protocols can identify linear behavior. This experiment differed from the FSM experiment primarily by the fact that the sine wave signal had a zero volt offset. The shaker amplifier potentiometer was adjusted to give the sine wave a $10 \mathrm{~N}$ amplitude.

The FSM experiment tested the pin-clevis joint in the fully deployed position (see Figure 8 ). The joint node was fixed in place by a joint mounting bracket, which is assumed to prevent all motion by the joint node. The three faces that the lasers hit were covered with white paper tags to provide a relatively smooth, high contrast target surface.

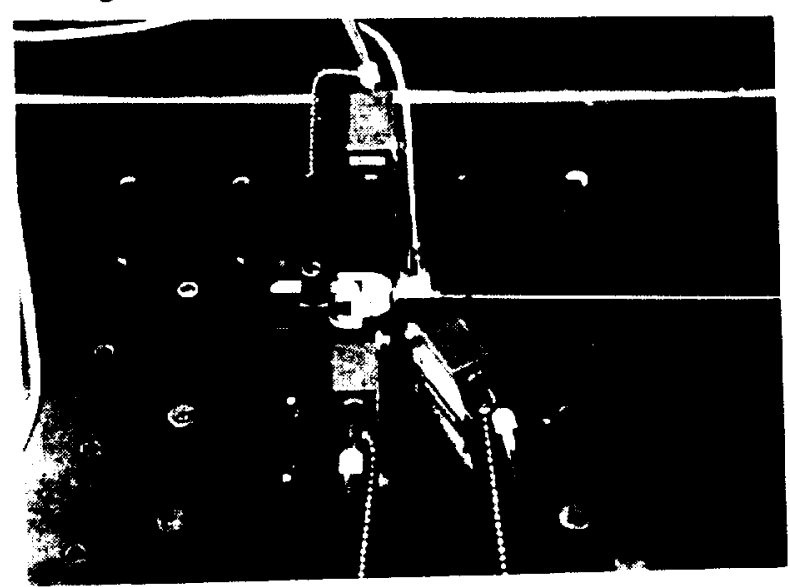

Figure 8: Testing Joint in Fully Deployed Position

\section{Analysis Protocol}

Three operations are performed on the data from each experiment before it is plotted. First the data is post-processed. The second data operation is to extract the motion of the desired states from the laser measurements. The last data operation is to form the vector triples that will define the three dimensional (3D) force-state map.

There are several phases to the data post-processing. The first involves removing the average offset value in each ensemble of each sensor. This is done for two reasons. First, the lasers cannot be mounted exactly such that their zero calibration value coincides with the steady-state position of their individual target fac- es. In addition, the motion of interest is that part of the motion which is greater than the steady-state response. The next postprocessing phase is the negating of all the values for each ensernble of the force transducer. This is done because FSM assumes that tensile loads have a positive value and the force transducer produces a negative voltage when put in tension. The last postprocessing phase is filtering the data with a forward and backward fourth order lowpass Butterworth filter with a comer tre quency of $6.0 \mathrm{~Hz}$. This is done because other general plots and spectral analyses have determined that no significant frequency content exists above $6.0 \mathrm{~Hz}$ for an experiment with a $0.31 \mathrm{~Hz}$ ex. citation frequency.

An iterative procedure based on Newton's Mcthod is ust'd 10 extract the desired states in the second data operation. The six DOF's of the joint rigid body are defined as the axial uanslation motion due to tension and compression $\left(X_{t}\right)$, the Iranslational motion that parallets the pin of the joint $\left(Y_{f}\right)$, the vertical transl:1tional motion $\left(\mathcal{Z}_{t}\right)$, the rotational motion about the stinger axis $\left(X_{r}\right)$, the rotational motion about the pin $\left(Y_{,}\right)$, and the rotational motion about the vertical axis $\left(Z_{r}\right)$. The desired measured state is the axial state, $X_{t}$, and secondarily, the major degree of lreedom in the pin-clevis joint, $Y_{r}$. The other four DOF's, it observed, alle due to compliance in the joint.

The primary assumption of all the equations uscd in this stige of the analysis is that the clevis (see Figure 3 ) remains rigid under all the applied loads. This means that the geometry of the unloaded clevis remains constant throughout any experiment. So the target locations of the lasers on the unloaded clevis can a dso be assumed constant. With this known geometry information, $X$, and $Y_{n}$ can be found from the three lasers measurements bascd on the following assumptions

The first assumption is that the torsion state, $X_{,}$, is always $z e$ ro. Its effect could not be accounted for in the derived equation if its behavior was known. All five of the other DOF's are assumed present in the measured laser data. This means llat it tive lasers were available, the equations used in the current analysis protocol could be used without modification to identily all live DOF's. However, since only three lasers are used in Uese cxpreiments, the behavior of two of the DOF's inust be assuned Based on the displacement resolution of the lasers (2 microms), the manufacturing tolerances of the pin-clevis joint, and the observed joint behavior, the other two translational states, $Y_{l}$ and $Z_{l}$. are always assumed to have zero motion. The above assumptions imply that the compliance angle state, $Z_{r}$ is the dhird stale thit the three lasers can determine.

The third data operation of forming the vector triples for 3.1 ) plotting requires the differentiation of the displacement incitsurements of extracted states. The differentiation is performed by a three-point central difference rule 


\section{EXPERIMENTAL RESULTS}

Both experiments reported here recorded data sets for ten ensembles. Analysis of the data showed clear evidence that the observed behavior was highly repeatable. The results presented below are only for the first ensemble of each experiment for the sake of brevity.

\section{Linear Beam Experiment}

The cantilevered beam experiment was designed to show that the experimental and analysis protocols could identify linear behavior in a structure. By design, the static behavior of the beam test article also included a load path dependency. The effective length of the beam is reduced by about $11 \%$ when the beam is in compression. This is due to the plate that is connected to the beam which allows the beam to be rigidly fixed (see Figure 6 ).

The experimental results of one tension-compression cycle are presented in Figure 9. The digitized data was filtered with a forward and backward second order lowpass Butterworth filter with a comer frequency of $1.0 \mathrm{~Hz}$ to isolate the static and dynamic responses of the bearn. The test data in Figure 9 is represented by the solid line. The error in the measurements due to the $2 \mathrm{mi}$ cron resolution of the lasers is shown by plotting the error bounds in Figure 9. The upper bound on the displacements is shown by the dashed-dot line, while the lower bound is shown by the dashed line. The plot clearly shows the nearly linear static behavior of beam and the higher stiffness of the beam in compression due to a shorter effective length.

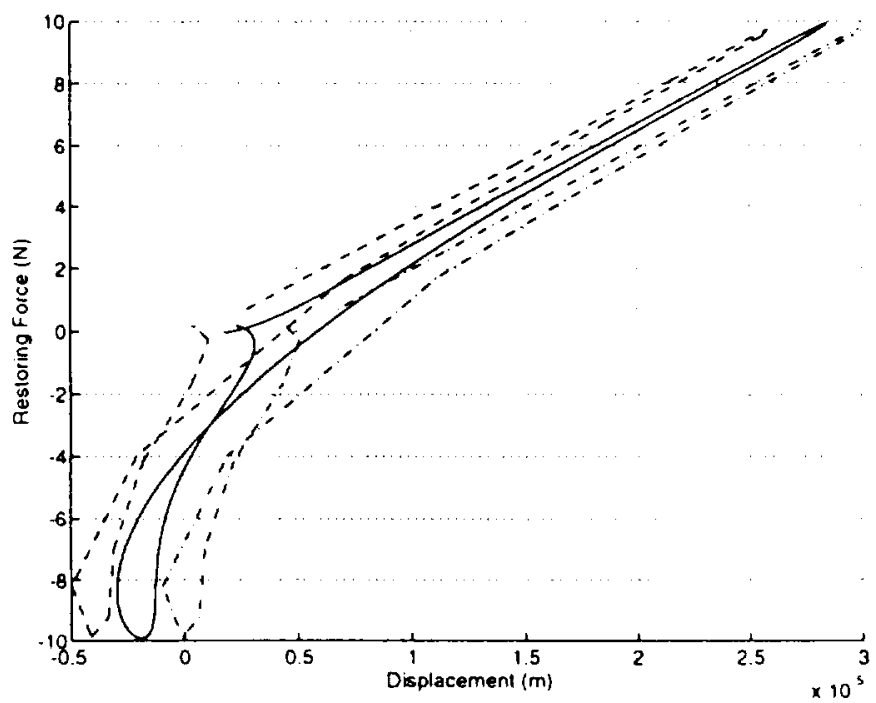

Figure 9: FSM of Beam with error envelope

\section{Nonlinear Joint Experiment}

The filtered digitized data for the pin-clevis joint from all four sensors are shown in Figures 10, 11, 12, and 13. The digitized data from the force transducer is shown in Figure 10. The displacement offsets have been removed from the laser measure- ments for the reasons mentioned above. The data in Figure 11 is from the laser that measures the motion of the angled lace of the pin-clevis joint. This laser is on the right in Figure 8 . The tiset on the left in Figure 8 measures the motion of side which has due hole for the pin in it. The digitized data for this second laser in shown in Figure 12. The verucal laser in Figure 8 meisures dhe motion of the top of the pin-clevis joint. This datat is presemted in Figure 13.

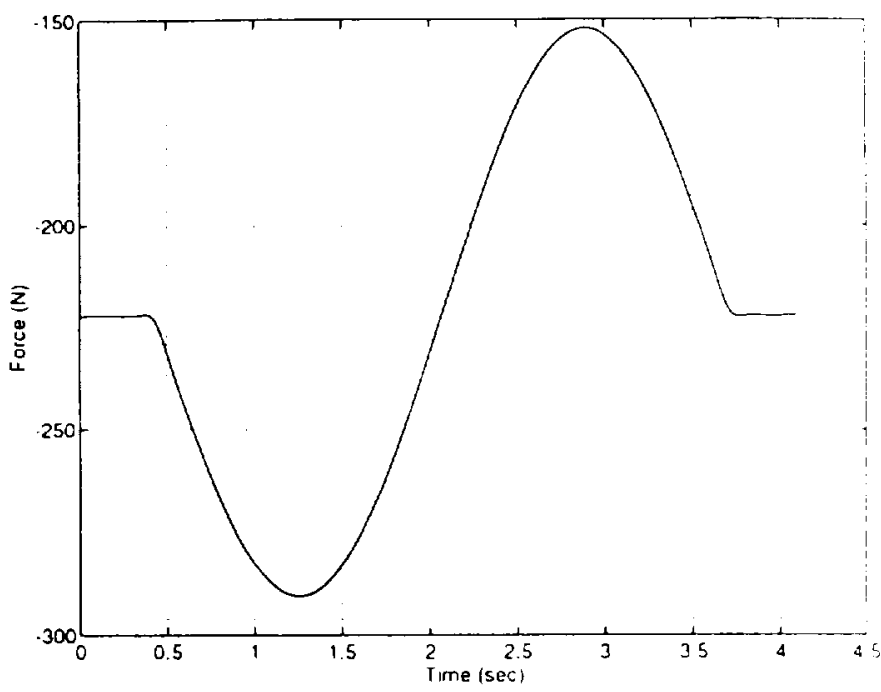

Figure 10: Filtered data from force transducer

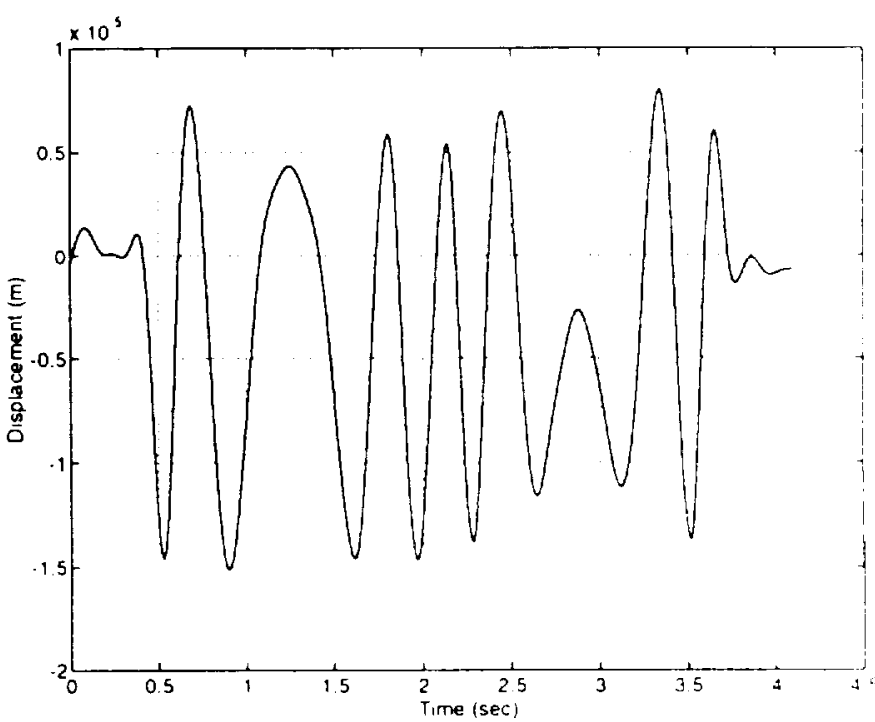

Figure 11: Filtered data from first laser

The force-state map of the ensile-compressive D()I: of the pin-clevis joint under a tensile preload is presconted in ligeure 14 The figure is shown only two dimensionally for llee satie of clatrity. The magnitude of the velocity state is quite how, langmen be. tween \pm 450 microns per second. If all the ditta allalys

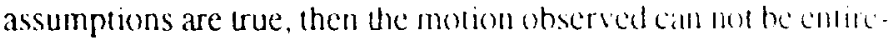
ly explained by elastic and shear deformations. The envile -compressive state, $X_{t}$, is observed to move about 30 microms atter the 
tensile load is increased by less than $20 \mathrm{~N}$. Then within the next $20 \mathrm{~N}$ of increasing tensile load, the state reverses direction and moves a total of over 40 microns. The clearest indication of the nonlinear nature of this behavior is that the restoring force is a multi-valued function of the displacement. This makes it impossible to generate a true FSM of the behavior because a single-valued surface can not be curve fitted to the data.

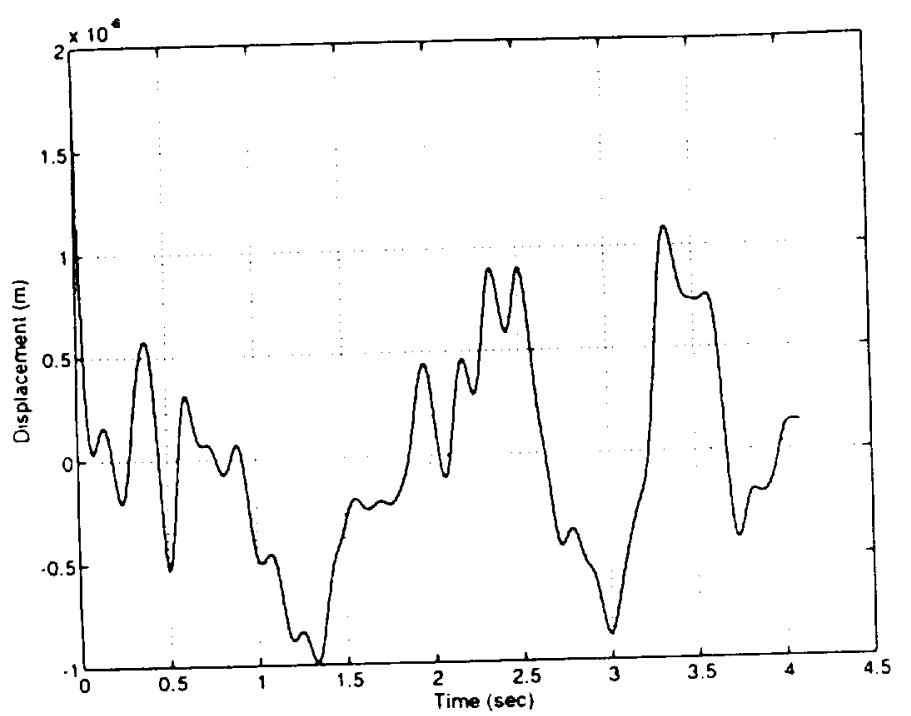

Figure 12: Filtered data from second laser

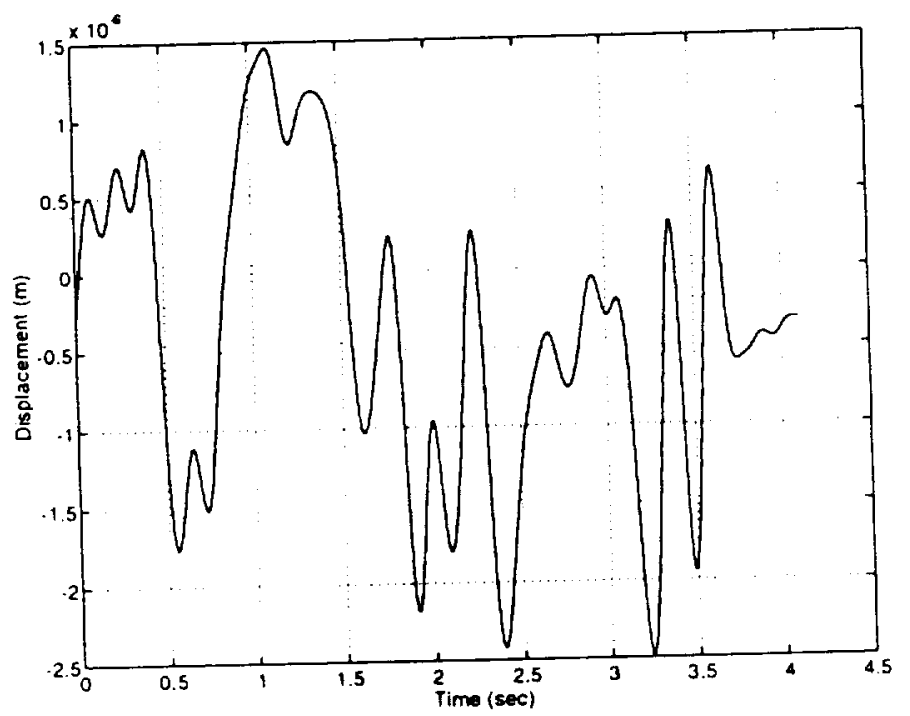

Figure 13: Filtered data from third laser

\section{CONCLUSIONS}

The experimental identification of micron-level nonlinear joint mechanics and dynamics for a class of joints found in prescision, adaptive, deployable space suructures is reported. The force-state mapping method is used to identify the behavior of a pin-clevis joint under a tensile preload and a tension-compression cycle at just one position along its deployment trajectory. The primary contribution of this work is the assessment that the micron-level behavior of pin-clevis joints under preloads is not always linear. Even under preloads, all the rigid body motion DOF's are highly coupled at micron levels of motion. The highly coupled behavior of all six DOF's in the pin-clevis joint prevent ed any meaningful nonlinear model from being developed tor the tensile-compressive state of the joint through the use of the force-state mapping technique.

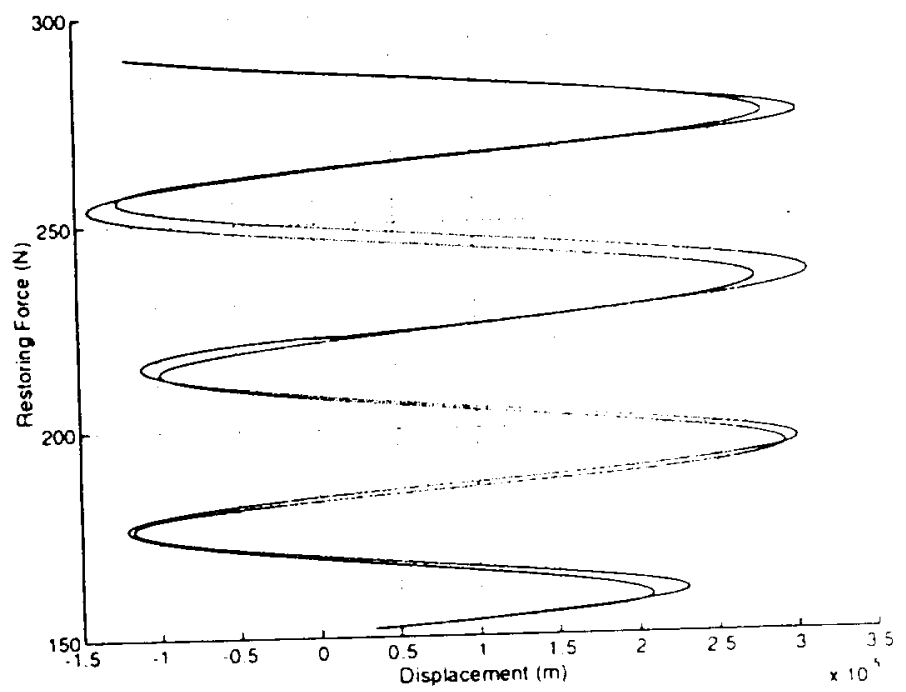

Figure 14: FSM of the tensile-compressive state, $X_{t}$

The highly nonlinear behavior observed places serious doubl on the validity of the other assumptions made in order fo extratc the joint states. Without strain gages, it would be difficult to (letermine if the joint is experiencing any elastic or shear detormattion. Without additional lasers and otler sensors, it was impossible to validate the assumed motion of the torsional, vertical translation, and transverse translation stiltes. The complex nonlinear experimental results indicate that all six rigid body DOF's of the pin-clevis joint need to be measured in order to accurately extract the tensile-compressive state. The results su:gest that at micron levels of motion modelling of the joint mechanics and dynamics must include the interactions between all internal joint components. The presence of internal components, such as a deformable pin, bushings, bearings, and surfice coatings, also implies that a pin-clevis joint can never be accurately modelled as a SDOF system for hehivior (nt the micton level.

\section{ACKNOWLEDGMENTS}

This work was supported by the Center lor Space Comstmic tion (CSC) through NASA Grant No. NAGW-1388 and by NASA Langley Research Center (LaRC) through NASA (inim No. NAG-1-1490. The first author has also been supporled in part by a National Science Foundation Graduate Fellowship. The authors would like to acknowledge the assistatke provided by Shannon Gaydos, an undergraduate research assistimt, in the de sign and assembly of the test equipment. 


\section{REFERENCES}

1. Masri, S. F. and Caughey. T. K., "A Nonparametric Identification Technique for Nonline as Dynamic Problems," Journal of Applied Mechanics, Vol. 46, 1979. pp. 433-447.

2. Crawley, E. F. and Aubert, A. C.. "Identification of Nonlinear Structural Elements by Force-State Mapping." AIAA Journal, Vol. 24, Jan. 1986, pp. 155-162.

3. Masri, S. F, Sassi, H., and Caughey, T. K, "Nonparametric Identification of Nearly Arbitrary Nonlinear Systems," Journal of Applied Mechanics, Vol. 49, 1982, pp. $619-627$.

4. Aubert, A. C., Crawley, E. F., and O'Donnell, K. J., "Measurement of the Dynamic Properties of Joints in Flexible Space Structures," M.S. Thesis, MIT Cambridge, MA, Sept. 1983.

5. Masters, B. P. and Crawley, E. F., "Multiple Degree of Freedom Force-State Component Identification," Proceedings of the 34th AIAAASME/ASCE/AHS/ASC Structures, Structural Dynamics and Materials Conference, La Jolla, April 1993, ALAA-93. 1654.

6. Worden, K., "Parametric and Nonparametric Identification of Nonlinearity in Structural Dynamics," Ph.D. Thesis, HeriotWatt University, Edinburgh, Nov. 1989.

7. Worden, K. and Tomlinson, G. R., "Parametric and Nonparametric Identification of Automotive Shock Absorbers," Proceedings of the 10th International Modal Analysis Conference, San Diego, Feb. 1992, pp. 764-771.

8. Rhodes, M. D., "Design Considerations for Joints in Deployable Space Truss Structures," Proceedings of the Ist NASA DOD CSI Technology Conference, Norfolk, Nov. 1986, NASA Conference Publication 2447, Part 1.

9. Timoshenko, S. P. and Goodier, J. N., Theory of Elasticity: Third Edition, McGraw-Hill Publishing Company, 1987.

10. Hinkle, J. D. and Peterson, L.D., "Experimental Dynamic Characterization of Reconfigurable Adaptive Truss," to appear in Proceedings of the 35ih AIAA/ASME/ASCEJAHS/ASC Strucitures, Siructural Dynamics and Materials Conference, Hilion Head, April 1994, AlAA-94-1734.

11. Al-Hadid, M. A. and Wright, J. R., "Developments in the ForceState Mapping Technique for Non-Linear Systems and the Ex tension to the Location of Non-Linear Elements in a Lumped. Parameter System," Journal of Mechanical Systems and Signal Processing, Vol. 3, 1989, pp. 269-290.

12. Al-Hadid, M. A. and Wright, J. R., "Application of the ForceState Mapping to the Identification of Nonlinear Systems," Paper presented at the European Forum on Aeroelasticity and Structural Dynamics, Aachen, 1989. 